\title{
Minimally Invasive Management of Fracture Calcaneum
}

\author{
${ }^{1}$ Mandeep S Dhillon, ${ }^{2}$ Sharad Prabhakar, ${ }^{3}$ Nitesh Gahlot, ${ }^{4}$ Bhava RJ Satish
}

\begin{abstract}
Modern usage of minimally invasive methods has been applied to fractures of the calcaneus with good effect in the 21st century. The Essex-Lopresti maneuver has been modified, distraction devices are being judicially used, and the advent of image intensifiers and small joint arthroscopes have allowed accurate visualization of the reduced fracture as well as joint surfaces. It is important to understand that the three-dimensional (3D) anatomy, Böhler and Gissane angles, and heel height have to be restored. The surgeon should be ready to convert to an open procedure at any time. We describe here the method we use in our institute, and this is one of the many methods of managing this complex injury.
\end{abstract}

Keywords: Calcaneus fracture, Kirschner wire fixation of calcaneus fractures, Minimally invasive surgery, Percutaneous reduction.

How to cite this article: Dhillon MS, Prabhakar S, Gahlot N, Satish BRJ. Minimally Invasive Management of Fracture Calcaneum. J Foot Ankle Surg (Asia-Pacific) 2016;3(1):47-52.

Source of support: Nil

Conflict of interest: None

\section{INTRODUCTION}

The treatment of calcaneal fractures has been a topic of controversy and discussion for a long time. Management protocols for calcaneal fractures have evolved from nonoperative treatment with bandaging and elevation, through open reduction and plate fixation, to minimally invasive surgery (MIS) at present. Bell performed the first documented open reduction in an open fracture in 1882, ${ }^{1}$ while Morestin in 1902 did open reduction and internal fixation (ORIF) for the first time by a lateral approach. $^{2}$

\footnotetext{
${ }^{1}$ Professor and Head, ${ }^{2}$ Associate Professor, ${ }^{3}$ Assistant Professor ${ }^{4}$ Chief Surgeon

1,2Department of Orthopaedics, Postgraduate Institute of Medical Education and Research, Chandigarh, India

${ }^{3}$ Department of Orthopaedics, All India Institute of Medical Sciences, Jodhpur, Rajasthan, India

${ }^{4}$ Department of Orthopaedics, BRJ Ortho Centre \& MAK Hospital, Coimbatore, Tamil Nadu, India

Corresponding Author: Sharad Prabhakar, Associate Professor, Department of Orthopaedics, Postgraduate Institute of Medical Education and Research, Chandigarh, India, Phone: +919781716119, e-mail: sharad.ortho@gmail.com
}

Currently, the most commonly used approach for ORIF of calcaneal fractures is the "extended lateral approach" which is the Letournel ${ }^{3}$ modification of the Palmer's approach. It provides an excellent exposure of the posterior facet and lateral wall of the calcaneus. ${ }^{3-9}$ The original lateral approach was given by Kocher, popularized later by Palmer, ${ }^{6}$ but results were not good due to wound sloughing and consequently a high infection rate.

Letournel modified the approach further by placing the incisions more posteriorly and inferiorly, elevating a full thickness skin flap so as to preserve the peroneal tendons, sural nerve, and the calcaneofibular ligament. Nevertheless a big problem with the open reduction of calcaneal fractures noted was the development of major wound complications owing to the vulnerable soft tissue envelope on the lateral aspect; angiosomes were recognized and the approach was further improved, with emphasis on full thickness flaps. Various studies report 0.4 to $14 \%$ incidence of wound edge necrosis and 1.3 to $7 \%$ infection rates with the extended lateral approach. ${ }^{9-15}$ Medial approaches carry the risk of neurovascular injury along with inadequate exposure and are limited to specific fractures primarily involving the sustentacular tali. The chances of wound necrosis increase to $27 \%{ }^{15}$ when combined medial and lateral approaches are used for ORIF.

\section{MINIMALLY INVASIVE CALCANEAL FRACTURE SURGERY}

Due to the soft tissue problems associated with open reduction of calcaneus fractures, the focus has shifted on to minimally invasive methods in the 21st century. Four basic types of minimal invasive methods have been described for managing displaced intra-articular calcaneus fractures:

1. Percutaneous reduction of fragments by Kirschner wire (K-wire)/Steinmann pin leveraging 2,16-19

2. Distraction by application of external fixators $\mathrm{s}^{20-22}$ and subsequent stabilization by percutaneous methods

3. Ligamentotaxis with subsequent percutaneous screw fixation $^{23,24}$

4. The sinus tarsi approach

The first three methods form the basis of this article. 
The method of indirect closed reduction by percutaneous leverage with a pin introduced percutaneously into the main tuberosity fragment was first done by Westhues in $1934 .{ }^{2,25}$ Gissane popularized this method in the United States. Essex-Lopresti, ${ }^{2}$ however, found it to be particularly useful in tongue-type fractures and described the maneuver which later became known by his name in English literature, ${ }^{19,26,27}$ while German articles still refer to it as the Westhues method. ${ }^{28-30}$ Tornetta ${ }^{26}$ modified the procedure by replacing pins with percutaneous screw, and Gavlik et al ${ }^{28}$ combined arthroscopy of the subtalar joint with percutaneous screw fixation of displaced calcaneus fractures to see reduction at the posterior facet.

\section{PREREQUISITE FOR CALCANEUS FRACTURE MIS}

Minimally invasive surgery is ideally done early, usually within 8 to 10 days of injury. The reason is that with passage of time the fracture consolidates, fracture margins become sticky and are not easy to manipulate, resulting in inadequate reduction and extensive struggles (Figs 1A to C).

\section{INDICATIONS FOR MIS CALCANEUM FRACTURES}

\section{Patient-related Factors}

- Systemically critical condition of the patient, to minimize the time of surgery, e.g., polytraumatized, multiply injured patients

- Contraindications to open reduction, e.g., severe, poorly controlled diabetes, heavy smokers, immunodeficiency

- Some bilateral cases.

\section{Soft-tissue-related Factors}

- Open fractures

- Blistering and superficially contaminated wounds

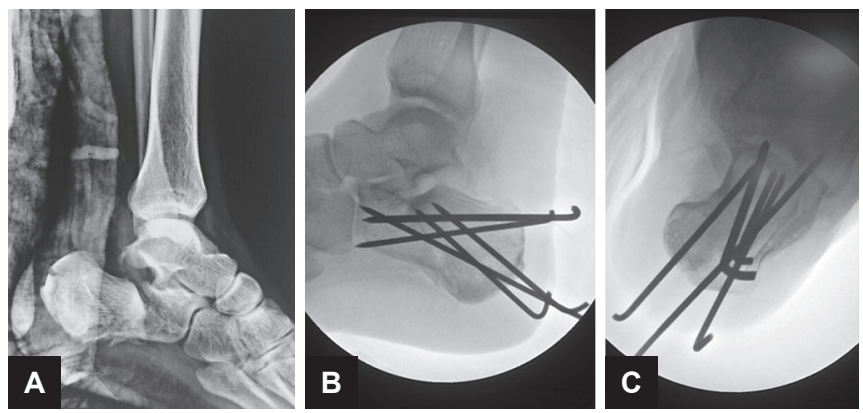

Figs 1A to $C$ : (A) Tongue-type fracture which is ideal for fracture fixation by percutaneous methods; ( $B$ and $C$ ) Percutaneous fixation by K-wires; note all wires are short of the joints
- Severe soft tissue vascular compromise

- Compartment syndrome of foot.

\section{Fracture Pattern Indications}

- Tongue-type fracture pattern

- Extra-articular fractures not involving weight-bearing posterior facet

- Sanders type IIc - the fracture line is far medial so that it leaves most of the posterior facet intact

- Sanders type IIa and IIb - need good view of intraarticular fracture reduction via arthroscopy or a small incision sinus tarsi approach

- Comminuted fractures - aim here is to achieve as much subtalar joint congruity as possible and maintain the heel height and calcaneus length by manipulating the major fragments. We may need to apply an external fixator to achieve the same.

\section{PRINCIPLES OF MANAGEMENT}

Minimally invasive surgery relies on the principle of ligamentotaxis and uses the strong joint capsule and ligaments surrounding calcaneum - e.g., talocalcaneal, calcaneofibular, tibiocalcaneal ligaments - to aid in reduction.

Four basic aims have to be achieved while operating calcaneus fractures:

1. Restore height and length of calcaneum

2. Correction of varus/valgus alignment of the tuberosity

3. Correction of heel widening and repositioning of lateral wall

4. Posterior facet anatomic, or near anatomic, reduction.

\section{SURGICAL PROCEDURE: HOW I DO IT}

The operating table should be radiolucent, and goodquality image intensifier is necessary like any MIS. In unilateral cases, patient is placed in the lateral position. In bilateral cases patient position is prone (as also in cases where concomitant spinal surgery is to be performed), and a cotton roll or sheet roll is placed below the distal tibia so that the knee is kept in partial flexion, allowing ankle and heel manipulations. The tourniquet is placed at mid-thigh level. If there is an open wound, it should be thoroughly debrided first; redraping and change of instruments is essential before starting the second procedure of internal fixation to avoid any cross-infection.

\section{Minimally Invasive Surgery Technique for Tongue-type Fractures}

This includes the extra-articular fractures in EssexLopresti classification and Sanders type IIc intra-articular fractures.

We give a stab incision and pass a 3.5-mm Steinman pin from superolateral to the Achilles tendon, directed 
toward the anteroinferior margin, along the long axis of bone; this pin/wire goes into the tongue fragment of the calcaneum. Another 3.5-mm Steinman pin can be inserted at the posterior aspect of the calcaneum. The position of these is confirmed by image intensifier. Fracture reduction is performed by manipulating these two pins (principles used by Essex-Lopresti for his original maneuver) (Figs $2 \mathrm{~A}$ to $\mathrm{C}$ ). Simultaneous disimpaction and elevation of the posterior fragments is achieved. If the tongue fragment is small or bone is osteoporotic instead of 3.5-mm Steinman pins, $2.5-\mathrm{mm}$ $\mathrm{K}$-wires can be used. The above maneuver is useful in tongue-type fractures, particularly Sanders type IIc, when the posterior facet is intact and is not comminuted. However, it can also be used in other fracture subtypes along with additional maneuvers. If heel widening is present, indicating mediolateral displacement of fragments, an additional reduction clamp is placed with tongs on either side of heel, and gently tightened, compressing the fragments. The tongs should be ball-tipped so that it is not submerged into the bone upon tightening. Angulated ball-tipped clamps used in pelvic fracture fixation can be handy.
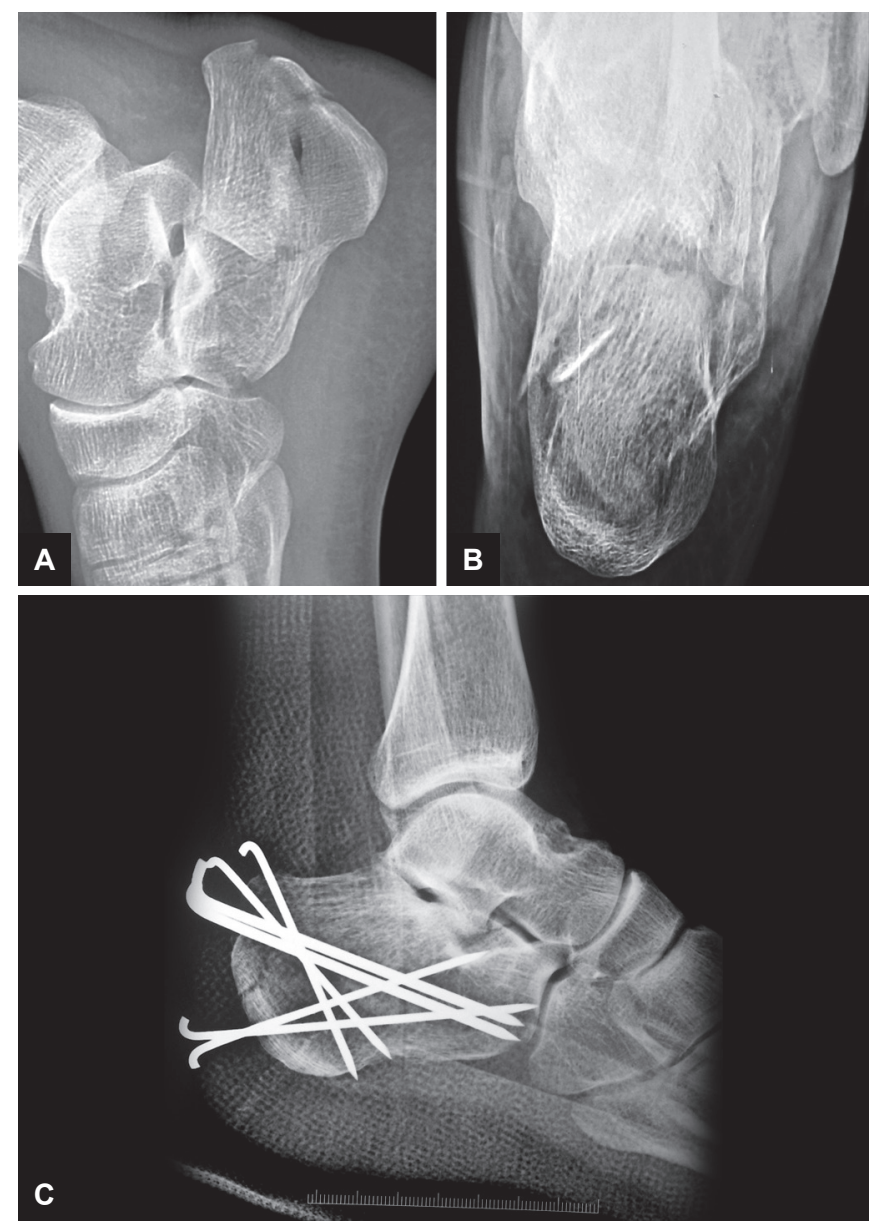

Figs 2A to C: (A and B) Lateral and axial views of complex calcaneus fracture; and (C) Postoperative lateral radiograph showing reduced fracture
Once fracture reduction is achieved, inserting multiple K-wires from the tuberosity into the anterior process stabilizes the fragments. Two K-wires are introduced from posterior to anterior one on medial side another on lateral side of Achilles tendon. One or two K-wires are introduced from plantar aspect toward the posterior facet. Routine transfixation of subtalar and calcaneocuboid joints is not required when the anterior process is intact and gives good purchase for K-wires. If calcaneocuboid joint is involved by fractures extending distally, then K-wires need to be advanced into cuboid and even cross the subtalar joint into the talus for additional stability.

A distractor or external fixator can be used to aid in reduction, and this can be used both in the vertical plane (from the mid portion of tibia shaft to the calcaneum) or horizontally in the plane of the foot (from two calcaneal pins at the posterior aspect, two either at the base of first metatarsal or at the lateral aspect of foot in the base of fifth metatarsal). With the simultaneous use of the distracters in two planes, the medial and lateral borders of foot are lengthened, along with restoration of heel height by longitudinal distraction, and the Böhler angle can be restored.

\section{Minimally Invasive Surgery Technique for Joint Depression Fractures}

This fracture pattern includes the Sanders type IIa, IIb, III, and IV fractures.

The initial procedure is same, with slight modifications. The direction of K-wire inserted in calcaneal tuberosity is from posterior-lateral to anterior-medial to correct the varus angulation of heel, which is common due to the displacement of the tuberosity.

We elevate the depressed lateral joint fragment under the $\mathrm{C}$-arm by a passing a bone punch inserted either from below through heel (Figs 2A to $\mathrm{C}$ ) or from lateral side through a separate small incision next to the peroneal tendons. Once the articular depression is corrected, the widened body of calcaneus is compressed side to side by a flat clamp. It is very important to reduce articular fragments first, otherwise they become impacted in displaced position. Threaded K-wires are passed (usually two) from the posteroinferior surface and often cross the subtalar joint, fixing the posterior facet fracture to the talus. Another two K-wires are then passed parallel to the body of the calcaneum, just beneath the fact fragment, anteriorly till the calcaneocuboid. These two K-wires are very important for the stability. These four K-wires form a three-dimensional (3D) grid construction below the posterior facet and provide good stability avoiding late collapse of depressed fragment. The K-wires can even be replaced by cannulated screws and would act as raft screws to prop up the depressed fragment. These wires are usually left in situ till bony union is visible. Distractors 

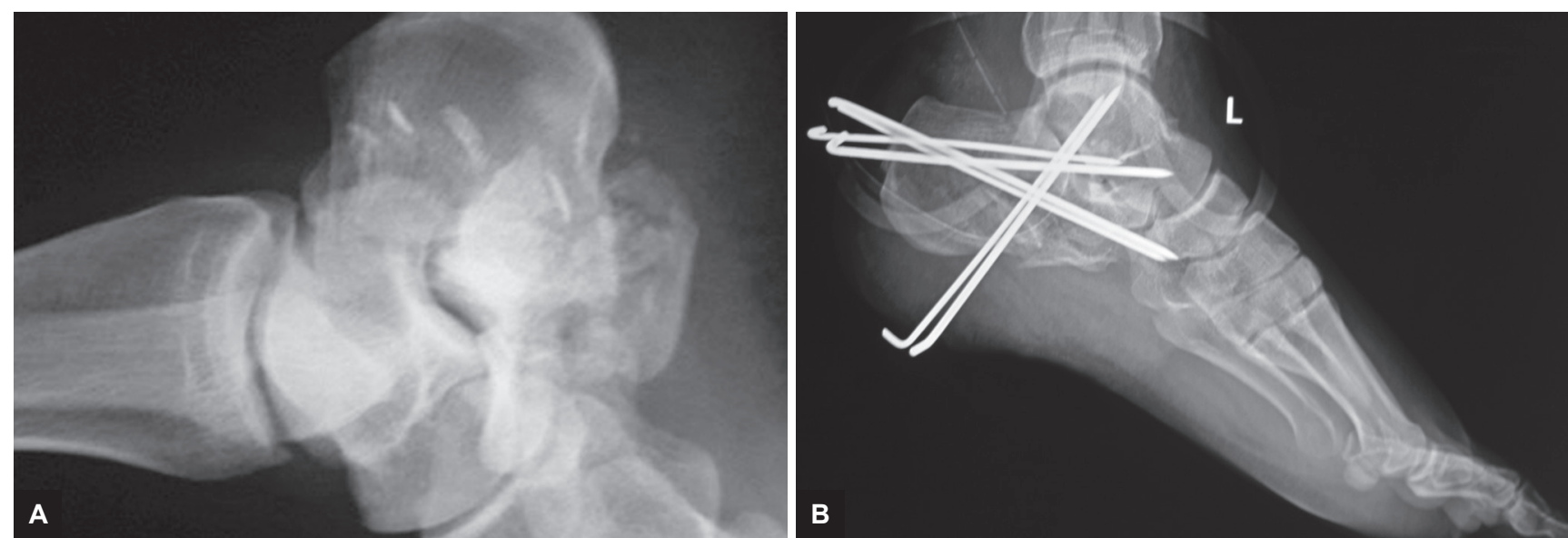

Figs 3A and B: (A) Comminuted complex fracture in a polytrauma patient; note the severe displacement, comminution, and change in Böhler angle; (B) Same case after MIS/percutaneous fixation; the reduction is acceptable and heel shape is regained. K-wires are across the joints to add to the stability of the construct

are applied along the medial and lateral borders when there is significant comminution, and stability is not very well achieved with the help of K-wires alone. It is important to note that one should not hesitate to cross both the subtalar or calcaneocuboid joints in cases of excessive comminution or instability. This is a temporary measure and seems not to disrupt function subsequently (Figs $3 \mathrm{~A}$ and B).

The use of screws may be an additional benefit. Compression screw can be inserted if the reduction is achieved by a small sinus tarsi approach and helps keep the joint fragment reduced and stable (Fig. 4). The depressed lateral fragment of the posterior facet can be maintained in reduced position by using partially threaded screws from lateral aspect toward sustentaculum tali. Longitudinal screws can be added by removing the K-wires. Cannulated screws can also be used as "raft screws" to support the facet fragment.

The key point in any MIS is the use of two pins inserted initially as joy sticks for reduction and manipulation,

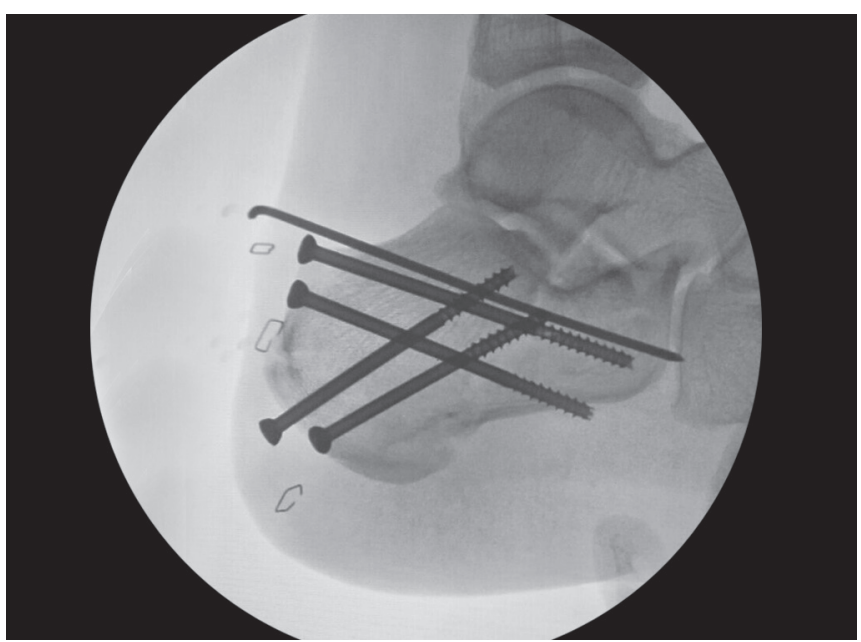

Fig. 4: Percutaneous reduction and fixation with screws and K-wires and intraoperative radiographs are the best guides for reduction. Lateral, axial, and broden views taken repeatedly are key factors for achieving and maintaining good reductions. The use of a distractor makes things easier, and many external fixators Joshi's External Stabilisation System (JESS) allow compression and distraction of the fragments along the vertical and horizontal plane and can subsequently be used as the definitive treatment modality. An example of stepwise reduction is shown in Figs 5A to I.

\section{POSTOPERATIVE REGIMEN}

Range of motion of ankle (and subtalar joints, if not transfixed) can be started on postoperative day two in simple noncomminuted fractures. Kirchner wires are kept for 8 to 10 weeks until radiographic union occurs. The patient is kept nonweight bearing till then. We prefer to delay weight bearing in cases with comminution and multiple fragments, so that some consolidation occurs. This decreases the chances of late collapse of the tuberosity and development of malunions. We start full weight bearing at 3 months, after evaluating union. Bilateral cases are difficult to rehabilitate as the use of partial weight bearing is not possible till both fractures unite.

\section{DISCUSSION}

Fracture calcaneum is the most commonly injured and disabling one among the fractures in the foot bones. Since the introduction of computed tomographic scans, the understanding of pathoanatomy has improved significantly, and management options have become more refined. Nonoperative treatment suffices for undisplaced or minimally displaced $(<2 \mathrm{~mm})$ fractures, while ORIF is the standard option for significantly displaced intraarticular fractures. However, the outcome depends heavily on the condition of surrounding soft tissues, as well as on the surgical insult caused while reducing and fixing the fractures using standard ORIF principles. 

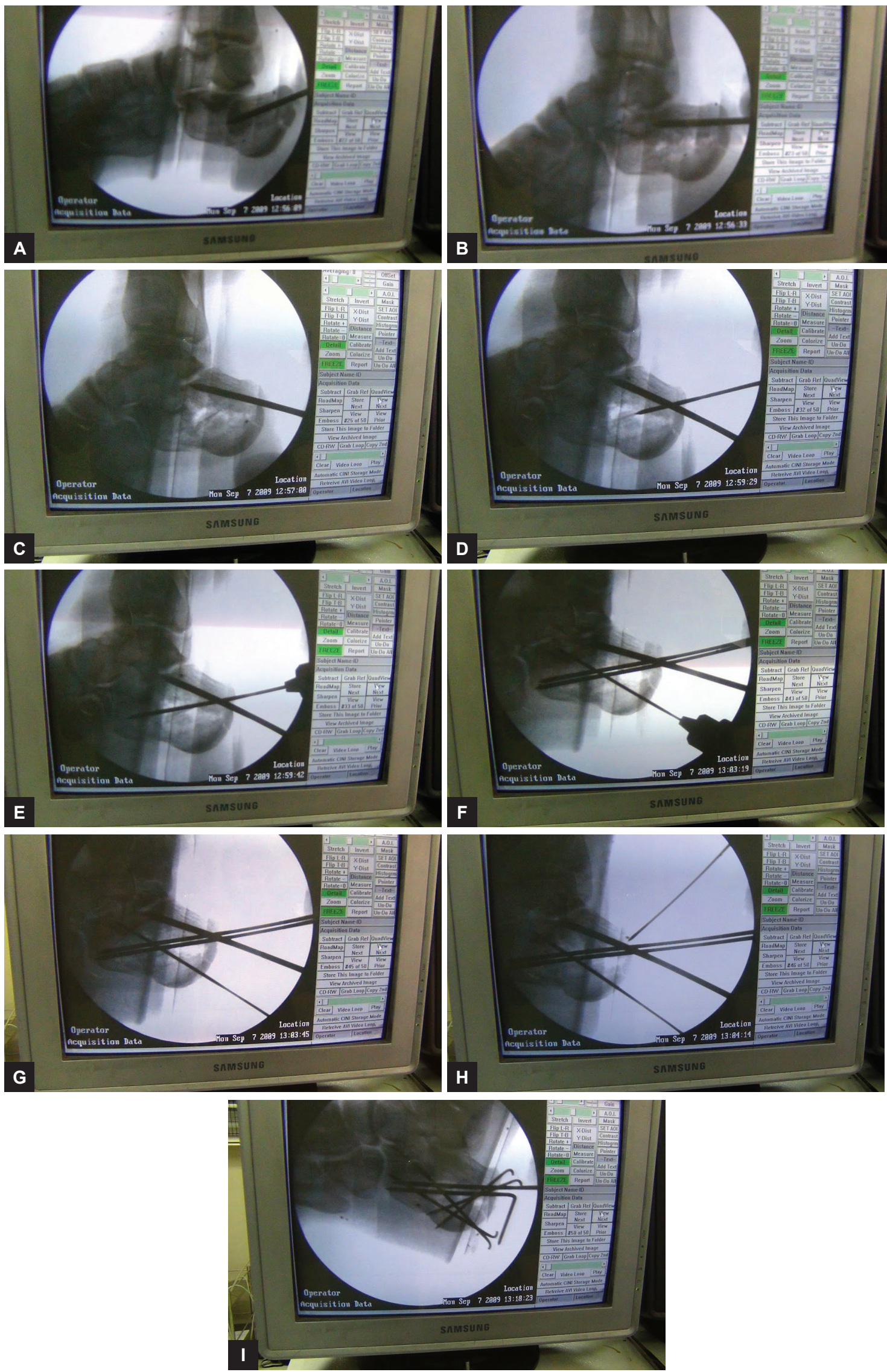

Figs 5A to I: (A) Insertion of $3 \mathrm{~mm}$ Steinmann pin into calcaneal tuberosity fragment; $(B, C)$ pin is levered down to restore tuberosity height and inclination; $(\mathrm{D}, \mathrm{E})$ tuberosity fragment is fixed to the anterior body using $2 \mathrm{~K}$-wires while reduction is maintained by holding the Steinmann pin; $(\mathrm{F}, \mathrm{G})$ the articular fragment is also fixed with $2 \mathrm{~K}$-wires; $(\mathrm{H})$ an additional K-wire is used to transfix the tuberosity fragment to the base of the calcaneus; and (I) final reduction 
Minimally invasive approaches have developed into good option for such cases; all extra-articular fractures, those with less comminution (Sanders type IIc), tongue type fragments, and even some calcaneus fractures which are a "bag of bones" may be reoriented to a near normal (3D) anatomy. The indications of MIS can be extended to Sanders type IIa, IIb, III, and selected type IV fractures, and can be better performed with the assistance of subtalar arthroscopy, or multiple small incisions to confirm articular reduction. However, the learning curve for MIS procedures is long, the instruments needed include an excellent image intensifier, maybe some arthroscopes, and distraction devices. The operating surgeon should be ready to convert MIS to open reduction intraoperatively without hesitation, if the acceptable articular reduction is not achieved.

\section{REFERENCES}

1. Goff CW. Fresh fractures of the os calcis. Arch Surg 1938;36(5): 744-765.

2. Essex-Lopresti P. The mechanism, reduction technique, and results in fractures of the os calcis. Br J Surg 1952 Mar;39(157):395-419.

3. Letournel E. Open treatment of acute calcaneal fractures. Clin Orthop Relat Res 1993 May;(290):60-67.

4. Bezes H, Massart P, Delvaux D, Fourquet JP, Tazi F. The operative treatment of intraarticular calcaneal fractures. Indications, technique, and results in 257 cases. Clin Orthop Relat Res 1993 May;(290):55-59.

5. Crosby LA, Fitzgibbons TC. Open reduction and internal fixation of type II intra-articular calcaneus fractures. Foot Ankle Int 1996 May;17(5):253-258.

6. Palmer I. The mechanism and treatment of fractures of the calcaneus; open reduction with the use of cancellous grafts. J Bone Joint Surg Am 1948 Jan;30A(1):2-8.

7. Sanders R. Displaced intra-articular fractures of the calcaneus. J Bone Joint Surg Am 2000 Feb;82(2):225-250.

8. Thordarson DB, Latteier M. Open reduction and internal fixation of calcaneal fractures with a low profile titanium calcaneal perimeter plate. Foot Ankle Int 2003 Mar;24(3):217-221.

9. Zwipp H, Tscherne H, Thermann H, Weber T. Osteosynthesis of displaced intraarticular fractures of the calcaneus. Results in 123 cases. Clin Orthop Relat Res 1993 May;(290):76-86.

10. Abidi NA, Dhawan S, Gruen GS, Vogt MT, Conti SF. Woundhealing risk factors after open reduction and internal fixation of calcaneal fractures. Foot Ankle Int 1998 Dec;19(12):856-861.

11. Benirschke SK, Kramer PA. Wound healing complications in closed and open calcaneal fractures. J Orthop Trauma 2004 Jan;18(1):1-6.

12. Benirschke SK, Sangeorzan BJ. Extensive intraarticular fractures of the foot. Surgical management of calcaneal fractures. Clin Orthop Relat Res 1993 Jul;(292):128-134.
13. Harvey EJ, Grujic L, Early JS, Benirschke SK, Sangeorzan BJ. Morbidity associated with ORIF of intra-articular calcaneus fractures using a lateral approach. Foot Ankle Int 2001 Nov;22(11):868-873.

14. Rammelt S, Barthel S, Biewener A, Gavlik JM, Zwipp H. Calcaneus fractures. Open reduction and internal fixation. Zentralbl Chir 2003 Jun;128(6):517-528.

15. Stephenson JR. Treatment of displaced intra-articular fractures of the calcaneus using medial and lateral approaches, internal fixation, and early motion. J Bone Joint Surg Am 1987 Jan;69(1):115-130.

16. Essex-Lopresti P. The mechanism, reduction technique, and results in fractures of the os calcis, 1951-52. Clin Orthop Relat Res 1993 May;(290):3-16.

17. King RE. Axial pin fixation of fractures of the Os calcis (method of Essex-Lopresti). Orthop Clin North Am 1973 Jan;4(1):185-188.

18. Nakaima N, Yamashita H, Tonogai R, Ikata T. A technique of dynamic reduction for displaced fractures of the thalamus of the calcaneum. Int Orthop 1983;7(3):185-190.

19. Tornetta P 3rd. The Essex-Lopresti reduction for calcaneal fractures revisited.J Orthop Trauma 1998Sep-Oct;12(7):469-473.

20. Magnan B, Bortolazzi R, Marangon A, Marino M, Dall'Oca C, Bartolozzi P. External fixation for displaced intra-articular fractures of the calcaneum. J Bone Joint Surg Br 2006 Nov;88(11): 1474-1479.

21. Schwall R, Junge RH, Zenker W, Besch L. Treatment of intraarticular calcaneus fractures with a para-articular external fixator. Unfallchirurg 2000 Dec;103(12):1065-1072.

22. Talarico LM, Vito GR, Zyryanov SY. Management of displaced intraarticular calcaneal fractures by using external ring fixation, minimally invasive open reduction, and early weightbearing. J Foot Ankle Surg 2004 Jan-Feb;43(1):43-50.

23. Levine DS, Helfet DL. An introduction to the minimally invasive osteosynthesis of intra-articular calcaneal fractures. Injury 2001 May;32(Suppl 1):SA51-SA54.

24. Rammelt S, Amlang M, Barthel S, Zwipp H. Minimallyinvasive treatment of calcaneal fractures. Injury 2004 Sep; 35(Suppl 2):SB55-SB63.

25. Rammelt S, Zwipp H. Calcaneus fractures: Facts, controversies and recent developments. Injury 2004 May;35(5):443-461.

26. Tornetta P 3rd. Percutaneous treatment of calcaneal fractures. Clin Orthop Relat Res 2000 Jun;(375):91-96.

27. Stein $H$, Rosen N, Lerner A, Kaufman H. Minimally invasive surgical techniques for the reconstruction of calcaneal fractures. Orthopedics 2003 Oct;26(10):1053-1056.

28. GavlikJM,RammeltS,ZwippH.Percutaneous, arthroscopicallyassisted osteosynthesis of calcaneus fractures. Arch Orthop Trauma Surg 2002 Nov;122(8):424-428.

29. Buch J. Bohrdrahtosteosynthese des Fersenbeinbruches. Akt Chir 1980;15:285-296.

30. Böhler L. Fersenbeinfrakturen. In: Böhler L, editor. Die Technik der Knochenbruchbehandlung. Wien: Maudrich; 1957. p. 2148-2217. 